\title{
Improved Hairpin Resonator for Microfluidic Sensing
}

\author{
Chia-Feng Liu, ${ }^{1}$ Ming-Kun Chen, ${ }^{1}$ Min-Haw Wang, ${ }^{2}$ and Ling-Sheng Jang ${ }^{1 *}$ \\ ${ }^{1}$ Department of Electrical Engineering, National Cheng Kung University, \\ East Dist., Tainan, 70101, Taiwan \\ ${ }^{2}$ Department of Electrical Engineering, Chinese Culture University, \\ Shilin Dist., Taipei, 11114, Taiwan
}

(Received December 4, 2017; accepted March 13, 2018)

Keywords: hairpin resonator, microwave sensor, microfluidic, ethanol, $\mathrm{NaCl}$

We present a near-field microwave sensor based on a hairpin resonator for detecting aqueous ethanol and aqueous $\mathrm{NaCl}$ concentrations in microfluidics. A hairpin sensor with a high-quality (high-Q) dielectric resonator allows the detection of a small variation in the aqueous $\mathrm{NaCl}$ concentration by measuring the scattering parameter $\left(S_{21}\right)$ responses at resonance frequencies. The responses caused by the test liquids with various dielectric constants in aqueous ethanol solution were observed at operating frequencies of 1.9 and $2.1 \mathrm{GHz}$. The detection of various concentrations of $\mathrm{NaCl}$ in solution was also achieved. The changes in $S_{21}$ at resonance frequencies are directly related to changes in the aqueous $\mathrm{NaCl}$ concentration because of the electromagnetic interaction between the resonator and the $\mathrm{NaCl}$ solution. The stable linear relationships are advantageous for detecting and analyzing aqueous ethanol and aqueous $\mathrm{NaCl}$ concentrations. It shows that the sensor is reliable for both dielectric constant and conductivity detection in liquid. The hairpin resonator, which is easily fabricated by standard printed circuit board (PCB) technology, has great potential for microfluidic sensing applications.

\section{Introduction}

Impedance measurement is one of the most promising techniques for developing label-free, real-time, and noninvasive methods of detection. Many sensors have been built for the detection of biotissues, and fluid concentrations such as those in biological cells, glycated hemoglobin $\left(\mathrm{HbA}_{1 \mathrm{c}}\right)$, glucose solution, and $\mathrm{NaCl}$ solution. ${ }^{(1-8)}$ However, at low frequencies, the contact area of the electrode-solution, tissue-solution, and tissue-electrode interfaces produces the electric double-layer capacitance effect, which influences the measured impedance. ${ }^{(9,10)}$ It is difficult to obtain the precise properties of biomaterials at low frequencies. In the low frequency range, the electrical response of the system is dominated by electrical double layer capacitance. In order to analyze and verify the measurement results obtained at low frequencies, the surface-specific double-layer capacitance effect must be included in the equivalent circuit model. It is difficult to obtain the precise properties of biotissues or medical indices. Moreover, at frequencies above $100 \mathrm{MHz}$, the interaction of microwaves and biological tissues is almost exclusively dependent

${ }^{*}$ Corresponding author: e-mail: 1sjang@ee.ncku.edu.tw http://dx.doi.org/10.18494/SAM.2018.1801 
on the aqueous and ionic content. It is very useful to have accurate values of the properties of biological tissues at these frequencies. ${ }^{(11)}$

Microwave sensing technology has been successfully used for measurement and detection. Many research groups designed sensors that operate at microwave frequencies to reduce the electric double-layer capacitance effect and enhance the accuracy of detection. A coplanar waveguide probe was built to perform single-HeLa-cell impedance measurements over the frequency range of $1.0 \mathrm{MHz}$ to $1.0 \mathrm{GHz} .^{(12)}$ Impedance sensors with nanometer gaps were prepared for the detection of biomolecular interactions such as protein-antibody and proteinaptamer binding. ${ }^{(13)}$

In addition, microwave resonant structures are used for developing noninvasive detection techniques. The microwave resonator, which is widely used for communication systems, is the most suitable microwave component for building a biosensor. ${ }^{(14,15)}$ A high-Q resonator can be applied to achieve high-sensitivity detection at a specific frequency. Many sensors composed of microwave resonators were designed to detect the electromagnetic wave interaction with water-based substances or biological materials. A planar microwave filter was utilized for biological cell discrimination by impedance spectroscopy analysis at microwave frequencies. ${ }^{(16)}$ A method of biomolecule detection where a double split-ring resonator excited by a microstrip transmission line is used was proposed. ${ }^{(17)}$ An electromagnetic resonant spiral sensor was designed for noninvasive D-glucose detection. ${ }^{(18)}$ An electromagnetic wave sensor operating in the 0.01 to $4.0 \mathrm{GHz}$ range for real-time chloride concentration analysis was reported. ${ }^{(19)}$ Thus, microwave technology has great potential for biosensing applications.

In the field of microwave communication technology, many types of resonator, such as end-coupled resonators, parallel-coupled resonators, interdigital coupled-line resonators, and dielectric resonators, are used for building microwave filters. ${ }^{(20-22)}$ However, the circuit size of conventional planar microwave resonators is large. A greater volume of the liquid under test (LUT) for measurement is required. In this study, hairpin resonators are first used for microfluidic sensing. We use a near-field microwave filter to construct a miniaturized hairpin sensor, which is half the size of a conventional hairpin resonator, for detecting aqueous $\mathrm{NaCl}$ concentration. ${ }^{(23)}$ The detection with only a $7.7 \mu \mathrm{L}$ volume of LUT was achieved. $\mathrm{NaCl}$ concentration is an important index in pathology, biology, food processing, and seawater detection. ${ }^{(24,25)} \mathrm{NaCl}$ in aqueous solution is often used as a standard liquid in experiments to measure the dielectric properties of biological material, because biological tissues typically have a high water content and a significant concentration of ions. ${ }^{(11)}$ In addition, different from the above studies that worked on single kind of liquid, this work performs detection of both aqueous ethanol and aqueous $\mathrm{NaCl}$ concentrations. The linear $S_{21}$ responses with dielectric constant and conductivity of liquid can be both achieved by the same hairpin sensor. Moreover, the microfluidic sensor with single-layer electrodes has many advantages: easily fabricated by standard PCB technology, low-cost, high reliability, fast response, and real-time detection. Thus, the hairpin resonator has great potential for microfluidic sensing applications. 


\section{Design and Fabrication}

\subsection{Design basis}

The spectra at $\mathrm{GHz}$ frequencies of deionized (DI) water and $\mathrm{NaCl}, \mathrm{KCl}, \mathrm{MnCl}_{2}$, and $\mathrm{CuCl}$ solutions of various concentrations were recorded with a microwave sensor. ${ }^{(19,26,27)}$ Radiation at microwave frequencies between 1.5 and $5.0 \mathrm{GHz}$ is only slightly attenuated by atmospheric gases. ${ }^{(28)}$ It has been observed that the frequency range of 0.5 to $4.0 \mathrm{GHz}$ is best suited for salinity estimation. ${ }^{(29)}$ The presence and concentration of various chloride solutions are also clearly evident in the changes in the amplitude and resonance frequency of the signal occurring especially at frequencies below 4.0 GHz. Moreover, for aqueous alcohols, the relative permittivity is dependent on the frequency. Above $2.2 \mathrm{GHz}$, as the frequency increases, the real part of complex permittivity decreases towards its high-frequency-limit relative permittivity value, and the imaginary part of complex permittivity increases to a peak value. ${ }^{(30)}$ According to the above, a microwave hairpin resonator sensor that operates at 1.9 and $2.1 \mathrm{GHz}$ for $\mathrm{NaCl}$ and ethanol detection, respectively, was designed in this work. The sensor is constructed of four hairpin-shaped half-wavelength stepped impedance resonators (SIRs) that create resonances as the sensing tool for the liquid detection, as shown in Fig. 1. ${ }^{(27)}$ Design parameters of the sensor are shown in Table 1. The SIR sensor is a transverse electromagnetic (TEM-) or quasi-TEM-mode resonator composed of two or more transmission lines with different characteristic impedances. Good resonance conditions at a specific frequency can be achieved by adjusting the impedance ratio. The specific resonance frequency is determined by the lengths and the characteristic impedances of the resonator. In order to further reduce the size of the resonator, the SIR structure was transformed into a hairpin shape. ${ }^{(31)}$

Table 1

Design parameters of the hairpin resonator sensor.

\begin{tabular}{lcrc}
\hline Symbol & Description & Value & Units \\
\hline$\varepsilon_{r}$ & Substrate dielectric constant & 3.38 & - \\
tan $\delta$ & Substrate loss tangent & 0.003 & - \\
- & Substrate thickness & 508 & $\mu \mathrm{m}$ \\
$W_{1}$ & Strip width & 3220 & $\mu \mathrm{m}$ \\
$W_{2}$ & Strip width & 2410 & $\mu \mathrm{m}$ \\
$W_{3}$ & Microstrip width & 580 & $\mu \mathrm{m}$ \\
$W_{4}$ & Microstrip width & 720 & $\mu \mathrm{m}$ \\
$W_{5}$ & Feed width & 1140 & $\mu \mathrm{m}$ \\
$g_{1}$ & Gap of resonators & 520 & $\mu \mathrm{m}$ \\
$g_{2}$ & Gap of coupled line & 460 & $\mu \mathrm{m}$ \\
$g_{3}$ & Gap of resonators & 610 & $\mu \mathrm{m}$ \\
$g_{4}$ & Gap of resonators & 1750 & $\mu \mathrm{m}$ \\
$L_{1}$ & Strip length & 8800 & $\mu \mathrm{m}$ \\
$L_{2}$ & Microstrip length & 10560 & $\mu \mathrm{m}$ \\
$L_{3}$ & Microstrip length & 5600 & $\mu \mathrm{m}$ \\
$L_{4}$ & Coupled line length & 5530 & $\mu \mathrm{m}$ \\
$L_{5}$ & Microstrip length & 7890 & $\mu \mathrm{m}$ \\
$L_{6}$ & Feed length & 1730 & $\mu \mathrm{m}$ \\
- & Copper thickness & 35 & $\mu \mathrm{m}$ \\
\hline
\end{tabular}




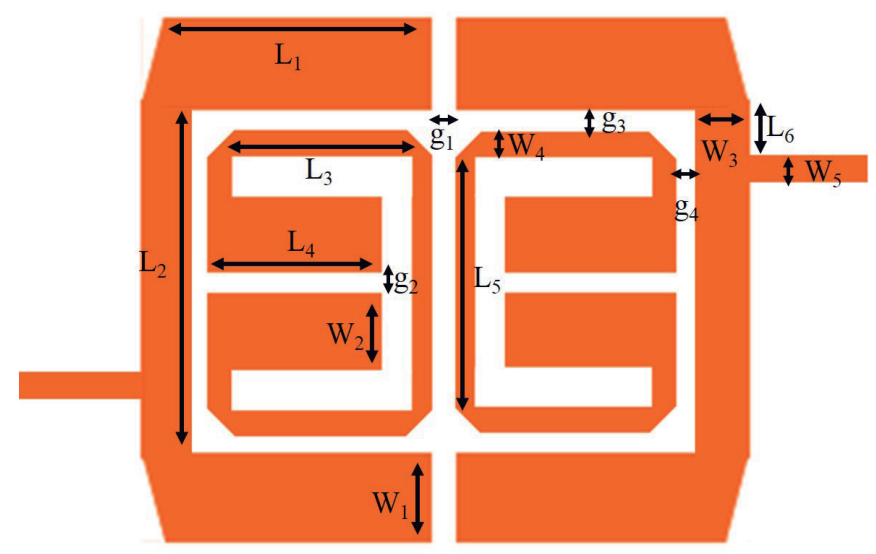

Fig. 1. (Color online) Geometry of the hairpin-shape resonator.

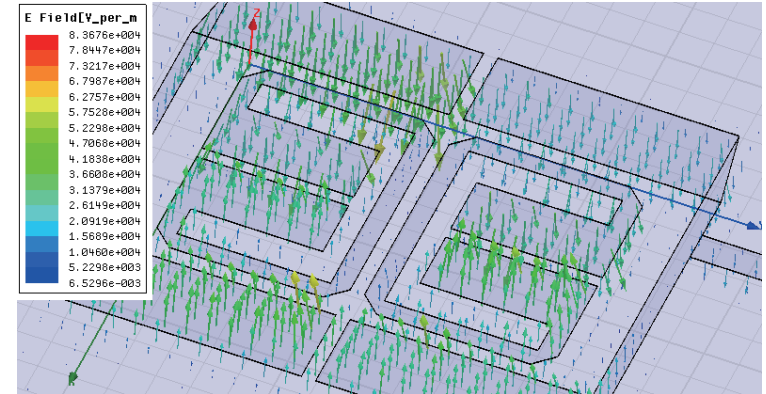

(a)

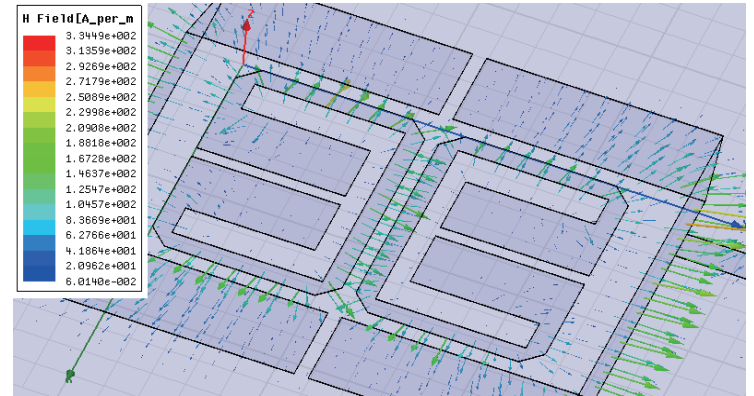

(b)

Fig. 2. (Color online) (a) HFSS model of the electric field distribution. (b) HFSS model of the magnetic field distribution.

Figure 2 shows the simulation results of the electric and magnetic field distribution. The crosstalk between resonators was observed. The scattering parameters are related to the mutual capacitor that represents the interaction between resonators. ${ }^{(23)}$ High sensitivity can be achieved by using the mutual capacitor as a sensing area. The mutual capacitor phenomenon was used to detect aqueous ethanol and aqueous $\mathrm{NaCl}$ concentrations.

\subsection{Fabrication}

The sensor was fabricated on a double-sided copper-clad Rogers RO4003C substrate with a thickness of $508 \mu \mathrm{m}$, a relative dielectric constant of 3.38, and a loss tangent of 0.0027 . The electrodes were fabricated using a PCB engraving machine. The bottom copper layer acted as the ground plane. Then, a subminiature version A (SMA) adapter was soldered onto the board, with the center pin directly connected to the signal line and ground pins connected to the back ground planes. Tygon R-3603 laboratory tubing (inner diameter: $0.7 \mathrm{~mm}$; outer diameter: 2.4 $\mathrm{mm}$ ) for containing the medium was bonded onto the sensor. Figure 3 shows the photograph 


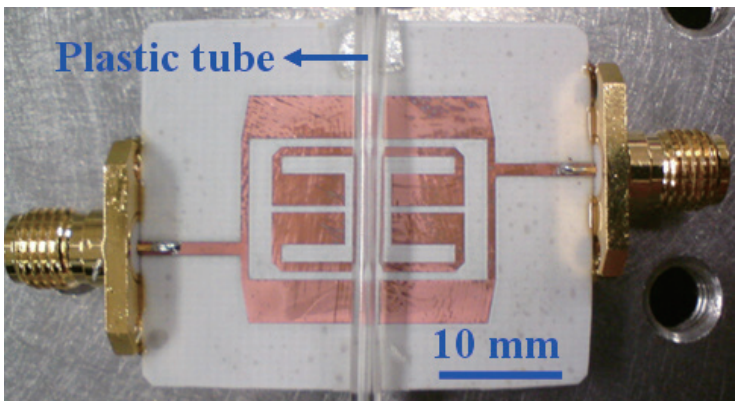

(a)

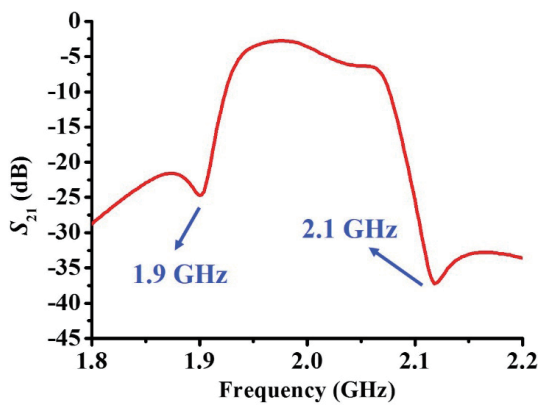

(b)

Fig. 3. (Color online) (a) Photograph of hairpin resonator sensor and (b) measured resonances of the sensor.

and measured $S_{21}$ responses of the sensor. The sensor has a center resonance at $2.0 \mathrm{GHz}$. The other two resonances occur at 1.9 and $2.1 \mathrm{GHz}$. These resonance conditions are suitable for the detection of liquid responses.

\section{Experimental Setup}

At microwave frequencies, systematic effects such as leakage, test port mismatch, and frequency response can affect measurement data. In a stable measurement environment, these effects are reproducible and can be eliminated using the vector network analyzer (VNA) with a calibration procedure. In accordance with the fixture and the VNA used in this work, shortopen-load-through (SOLT) calibration was used for this case. As shown in Fig. 4(a), a series of calibration structures, such as open, short, load, and through structures, were manufactured to improve the calibration accuracy. The $50 \Omega$ matched load was realized by two $100 \Omega$ SMD resistors connected in parallel to the ground plane, shown in Fig. 4(b). The calibration procedure was carried out by connecting a through kit, a load kit, a short kit, and an open kit to the desired test ports sequentially. All measurement instruments were controlled using a personal computer via a general-purpose interface bus line. The computer and custom-made software were used for automatic data acquisition and complex calculations.

The experiments were conducted at a temperature of $24 \pm 1{ }^{\circ} \mathrm{C}$ under a relative humidity of $43 \pm 10 \%$. The fluid was injected into the tubing using a syringe and allowed to travel to the outlet of the tubing. Then, the response of the fluid was measured by the VNA (Agilent Technologies E5062A) shown in Fig. 4(c).

\section{Results and Discussions}

\subsection{Aqueous ethanol concentration detection}

The measured $S_{21}$ responses of ethanol and DI water are shown in Fig. 5(a). Changes in $S_{21}$ caused by the medium can be observed at 1.9 and $2.1 \mathrm{GHz}$. Figures 5(b) and 5(c) show the detailed responses at 1.9 and $2.1 \mathrm{GHz}$, respectively. The measured $S_{21}$ at $1.9 \mathrm{GHz}$ increased 


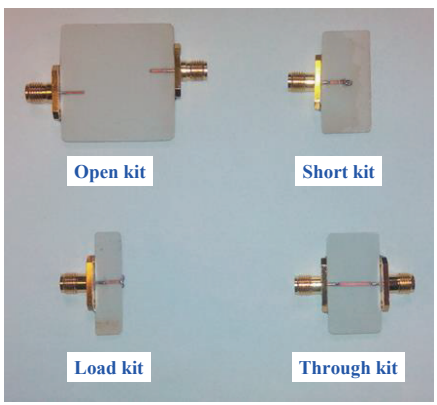

(a)

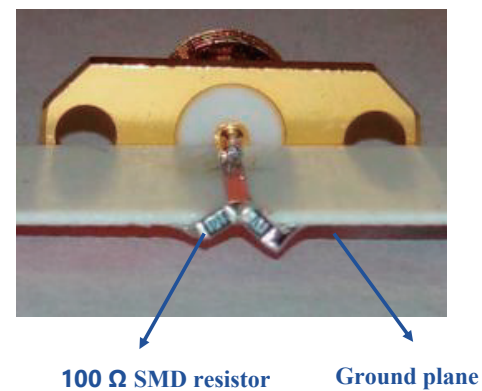

(b)

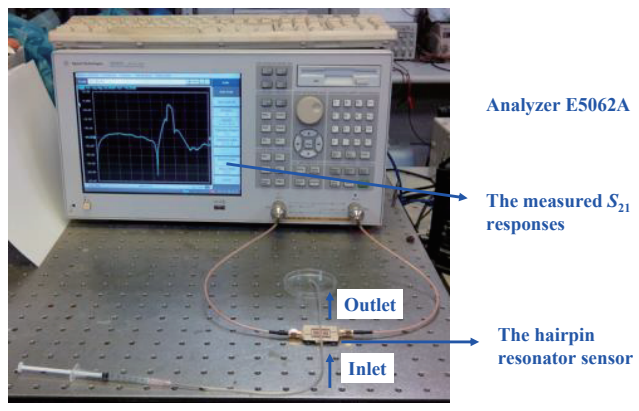

(c)

Fig. 4. (Color online) (a) The used calibration kits. (b) The load kit. (c) View of the measurement setup.

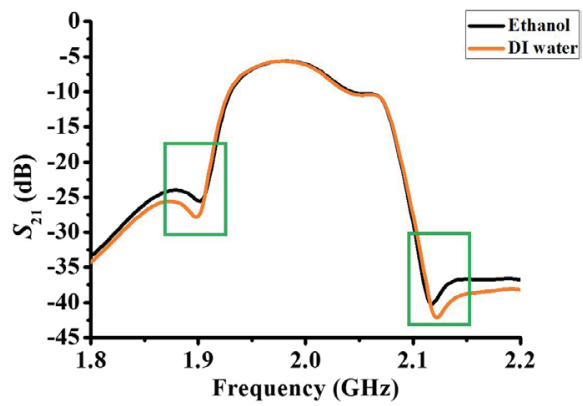

(a)

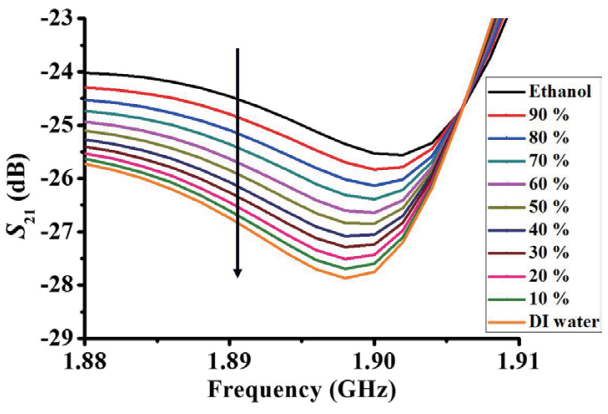

(b)

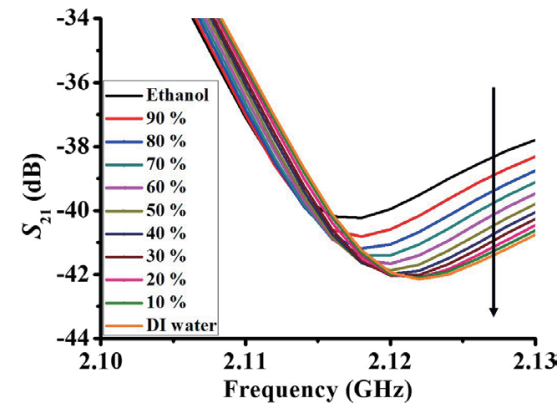

(c)

Fig. 5. (Color online) (a) Measured $S_{21}$ responses of ethanol and DI water. Detailed responses at (b) 1.9 and (c) 2.1 GHz. 
with increasing concentration of aqueous ethanol solution from $-27.9 \mathrm{~dB}$ for $0 \%$ to $-25.6 \mathrm{~dB}$ for $100 \%$. The measured $S_{21}$ at $2.1 \mathrm{GHz}$ also increased with increasing concentration of ethanol solution from $-42.1 \mathrm{~dB}$ for $0 \%$ to $-40.2 \mathrm{~dB}$ for $100 \%$. The dielectric constant of aqueous ethanol solution decreases with increasing ethanol concentration. ${ }^{(32)}$ According to coupled line theory, the coupling coefficient increases because of the larger capacitance between two coupled resonators. A high-dielectric-constant liquid directly causes the coupling capacitance to increase. At both resonances of 1.9 and $2.1 \mathrm{GHz}$, the larger dielectric constant of the LUT causes the greater attenuation. The variation of the dielectric constant of an LUT can be detected from the attenuation variation. Figure 6(a) shows the $S_{21}$ responses of aqueous ethanol concentrations from DI water to ethanol at intervals of $10 \%$. Regular changes in $S_{21}$ occurred at 1.9 and $2.1 \mathrm{GHz}$. The changes in $S_{21}$ at $1.9 \mathrm{GHz}$ with the variation of the percentage of aqueous ethanol solution are more linear than those at $2.1 \mathrm{GHz}$. At $1.9 \mathrm{GHz}$, the $S_{21}$ responses show a linearly increasing trend with increasing aqueous ethanol concentrations. The slope of the linear relationship between the $S_{21}$ responses and the aqueous ethanol concentration was found to be $0.023 \mathrm{~dB} / \%$.

The dielectric constants of ethanol, $80 \%$ aqueous ethanol solution, $50 \%$ aqueous ethanol solution, and water are known to be about 23.6, 35.4, 51.2, and 79, respectively. ${ }^{(32)}$ The measured $S_{21}$ and the trend line at $1.9 \mathrm{GHz}$ versus dielectric constant are plotted in Fig. 6(b). The coefficient of determination $\left(R^{2}\right)$ was found to be 0.9954 . The trend function is described by Eq. (1) where $S_{21, \text { measured }}$ is the measured $S_{21}$ at $1.9 \mathrm{GHz}$. Then the dielectric constant of aqueous ethanol solution $\left(\varepsilon_{r, \text { ethanol }}\right)$ can be derived as Eq. (2).

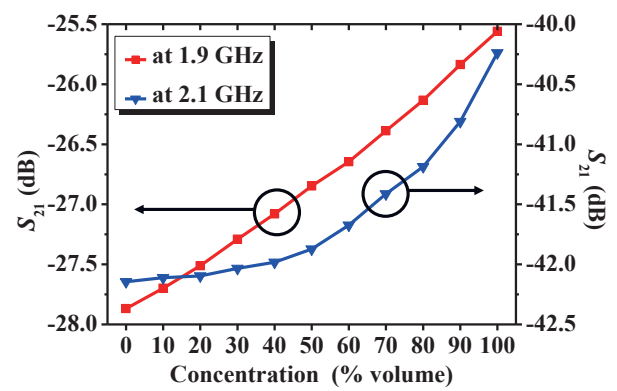

(a)

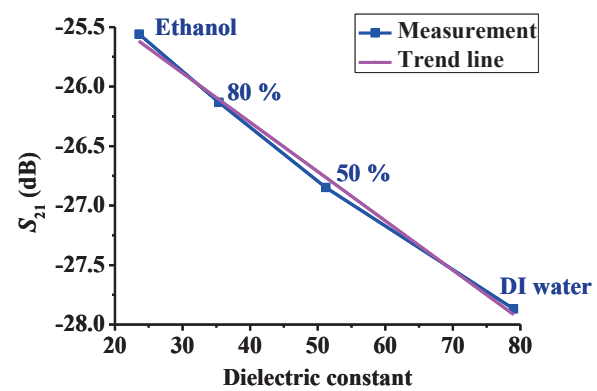

(b)

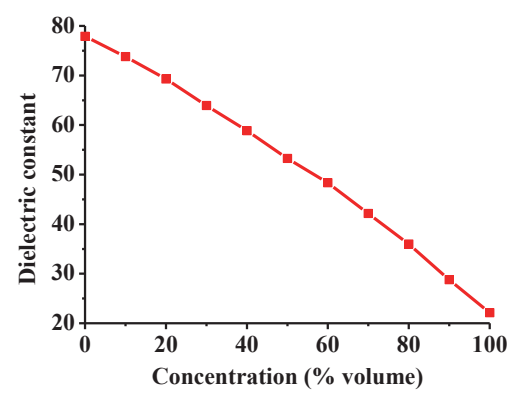

(c)

Fig. 6. (Color online) (a) Measured $S_{21}$ responses of aqueous ethanol concentrations from DI water to ethanol at 1.9 and $2.1 \mathrm{GHz}$. (b) Measured $S_{21}$ responses at $1.9 \mathrm{GHz}$ and the trend line plotted as a function of dielectric constant. (c) Estimated dielectric constant as a function of the aqueous ethanol concentrations. 


$$
\begin{gathered}
S_{21, \text { measured }}=-0.0414 \cdot \varepsilon_{r, \text { ethanol }}-24.644 \\
\varepsilon_{r, \text { ethanol }}=-\frac{S_{21, \text { measured }}+24.644}{0.0414}
\end{gathered}
$$

The estimated dielectric constant as a function of the aqueous ethanol concentration is plotted in Fig. 6(c). It shows that the sensor can be used to detect the ethanol content in aqueous solution through dielectric constant detection.

\subsection{Aqueous $\mathrm{NaCl}$ concentration detection}

The conductivities of aqueous $\mathrm{NaCl}$ concentrations from DI water to $100 \mathrm{mg} / \mathrm{mL}$ were measured using a conductivity meter (Suntex SC-110). As shown in Fig. 7(a), a linear relationship between the concentration and conductivity was observed. The conductivity of aqueous sodium chloride solution increases with increasing sodium chloride concentration. The conductivity of the LUT influences the $S_{21}$ responses because of the electromagnetic interaction between the sensor and the sodium chloride solution. Figure 7(b) shows the measured $S_{21}$ responses of aqueous $\mathrm{NaCl}$ solution of various concentrations. Figures 7(c) and 7(d) show the detailed responses at 1.9 and $2.1 \mathrm{GHz}$, respectively. Regular changes in $S_{21}$ occurred at 1.9 and 2.1

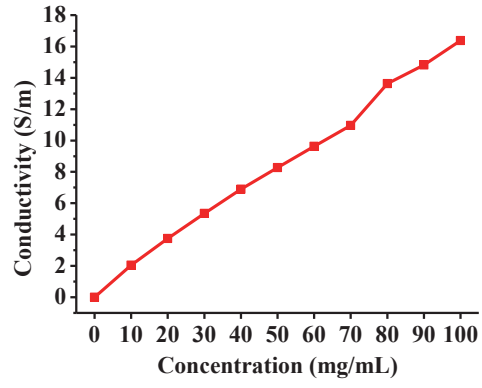

(a)

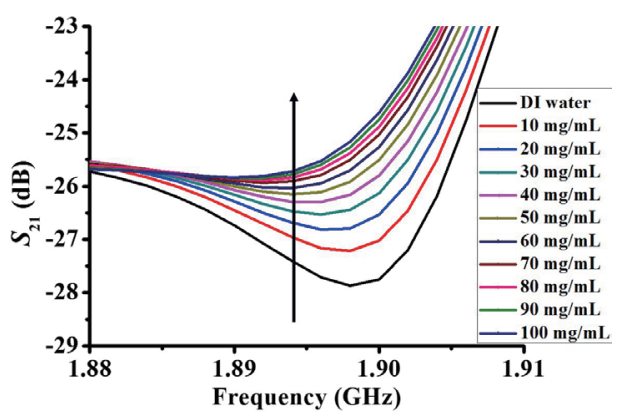

(c)

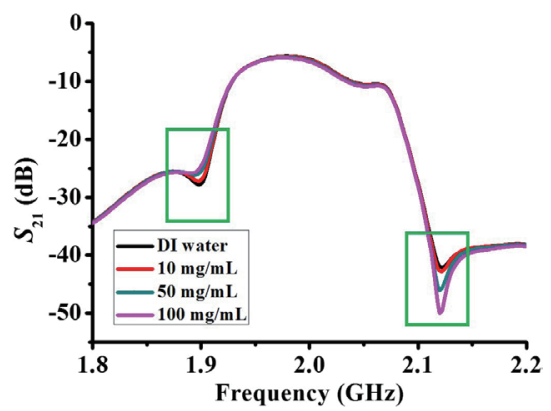

(b)

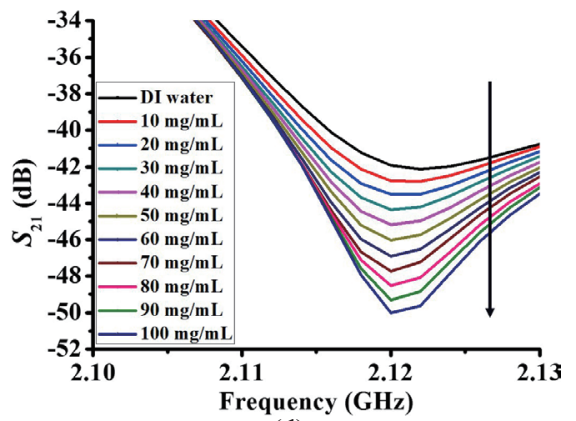

(d)

Fig. 7. (Color online) (a) Conductivity of aqueous $\mathrm{NaCl}$ solution with concentrations of 0 to $100 \mathrm{mg} / \mathrm{mL}$. (b) Measured $S_{21}$ responses of aqueous $\mathrm{NaCl}$ solution with various concentrations and DI water. Detailed responses at (c) 1.9 and (d) $2.1 \mathrm{GHz}$. 
GHz. The measured $S_{21}$ at $1.9 \mathrm{GHz}$ increased with increasing concentration of $\mathrm{NaCl}$ solution from $-27.9 \mathrm{~dB}$ for $0 \mathrm{mg} / \mathrm{mL}$ to $-25.8 \mathrm{~dB}$ for $100 \mathrm{mg} / \mathrm{mL}$. In contrast, the measured $S_{21}$ at 2.1 $\mathrm{GHz}$ decreased with increasing concentration of $\mathrm{NaCl}$ solution from $-42.1 \mathrm{~dB}$ for $0 \mathrm{mg} / \mathrm{mL}$ to $-50.0 \mathrm{~dB}$ for $100 \mathrm{mg} / \mathrm{mL}$. The greatest change in $S_{21}$ was about $8 \mathrm{~dB}$, which occurred at 2.1 $\mathrm{GHz}$ when the concentration was increased from 0 to $100 \mathrm{mg} / \mathrm{mL}$. The detection resolution in $\mathrm{mg} / \mathrm{mL}\left(R_{\text {detect }}\right)$ of the sensor is

$$
R_{\text {detect }}=\left|\frac{\Delta c}{\Delta S_{21}}\right| \times R_{\text {system }}
$$

where $\Delta c$ is the concentration variation in $\mathrm{mg} / \mathrm{mL}, \Delta S_{21}$ is the change in measured $S_{21}$ in $\mathrm{dB}$, and $R_{\text {system }}=0.05 \mathrm{~dB}$ is the resolution of the system. The detection resolution was found to be 0.63 $\mathrm{mg} / \mathrm{mL}$.

Moreover, the changes in $S_{21}$ at $2.1 \mathrm{GHz}$ are more linear than those at $1.9 \mathrm{GHz}$ for various concentrations shown in Figs. 8(a) and 8(b). At $2.1 \mathrm{GHz}$, the linear relationship between $S_{21}$ responses and aqueous $\mathrm{NaCl}$ concentration, $\left|\Delta S_{21} / \Delta c\right|$, was found to be $0.082 \mathrm{~dB} /(\mathrm{mg} / \mathrm{mL})$ in the range of 10 to $100 \mathrm{mg} / \mathrm{mL}$ and $0.069 \mathrm{~dB} /(\mathrm{mg} / \mathrm{mL})$ in the range of 0 to $10 \mathrm{mg} / \mathrm{mL}$.

In addition, the measured $S_{21}$ and the trend line at $2.1 \mathrm{GHz}$ versus conductivity are plotted in Figs. 8(c) and 8(d). The linear relationship between $S_{21}$ and conductivity suitable for low concentrations (1 to $10 \mathrm{mg} / \mathrm{mL}$ ) is described by Eq. (4). The sensitivity and $R^{2}$ were found to

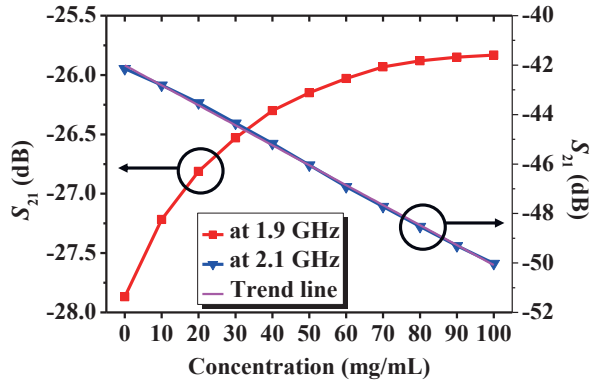

(a)

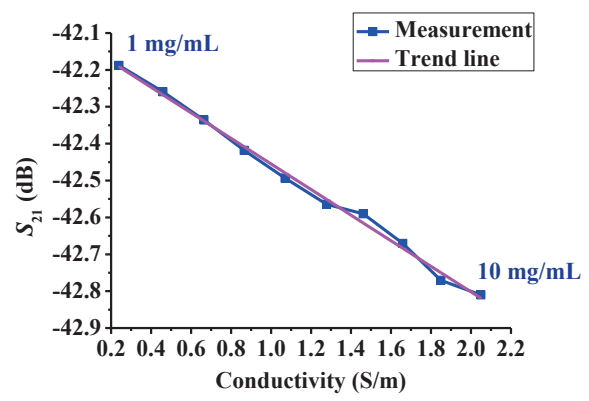

(c)

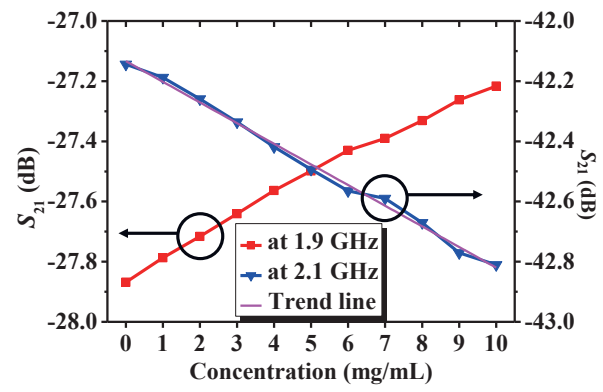

(b)

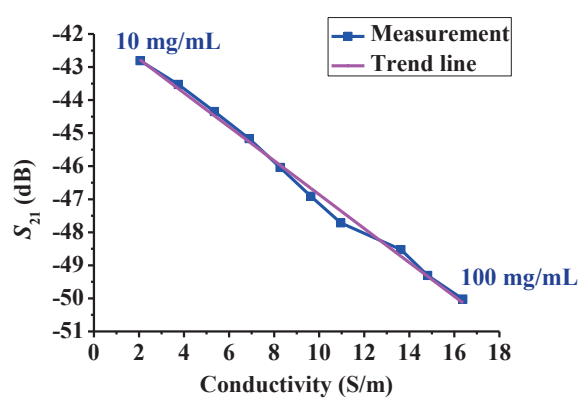

(d)

Fig. 8. (Color online) (a) Measured $S_{21}$ responses of aqueous $\mathrm{NaCl}$ concentrations ranging from 0 to $100 \mathrm{mg} / \mathrm{mL}$ and (b) from 0 to $10 \mathrm{mg} / \mathrm{mL}$ at 1.9 and $2.1 \mathrm{GHz}$. (c) Measured $S_{21}$ responses at $2.1 \mathrm{GHz}$ and the trend line plotted as a function of conductivity for the aqueous $\mathrm{NaCl}$ concentrations from 1 to $10 \mathrm{mg} / \mathrm{mL}$ and (d) 10 to $100 \mathrm{mg} / \mathrm{mL}$. 
be $0.3385 \mathrm{~dB} /(\mathrm{S} / \mathrm{m})$ and 0.9948 , respectively. Moreover, the linear relationship between $S_{21}$ and conductivity suitable for high concentrations (10 to $100 \mathrm{mg} / \mathrm{mL})$ is described by Eq. (5). The sensitivity and $R^{2}$ were found to be 0.5132 and $0.9956 \mathrm{~dB} /(\mathrm{S} / \mathrm{m})$, respectively, which are higher than those at low concentrations.

$$
\begin{gathered}
S_{21, \text { measured }}=-0.3385 \cdot \sigma_{\mathrm{NaCl}}-42.12 \\
S_{21, \text { measured }}=-0.5132 \cdot \sigma_{\mathrm{NaCl}}-41.729
\end{gathered}
$$

These stable linear relationships are helpful for detecting and analyzing aqueous $\mathrm{NaCl}$ concentrations. The sensor is thus suitable for biodetection applications.

\section{Conclusions}

We used miniaturized hairpin resonators with two resonances to determine aqueous ethanol and aqueous $\mathrm{NaCl}$ concentrations. The LUT was analyzed by observing the variation in scattering parameters at resonance frequencies. The $S_{21}$ was sensitive to the concentrations of aqueous ethanol and aqueous $\mathrm{NaCl}$ and had a linear relationship in the entire concentration range. The slope of the linear relationship between $S_{21}$ responses and aqueous ethanol concentrations at $1.9 \mathrm{GHz}$ was found to be $0.023 \mathrm{~dB} / \%$. A prototype sensor was used to demonstrate the measurement method for various concentrations of aqueous $\mathrm{NaCl}$. The detection resolution was $0.63 \mathrm{mg} / \mathrm{mL}$ with a $7.7 \mu \mathrm{L}$ volume at operating frequencies of 1.9 and $2.1 \mathrm{GHz}$. The slopes of the linear relationships between $S_{21}$ responses and aqueous $\mathrm{NaCl}$ concentrations at $2.1 \mathrm{GHz}$ were found to be $0.069 \mathrm{~dB} /(\mathrm{mg} / \mathrm{mL})$ in the range of 0 to $10 \mathrm{mg} / \mathrm{mL}$ and $0.082 \mathrm{~dB} /(\mathrm{mg} / \mathrm{mL})$ in the range of 10 to $100 \mathrm{mg} / \mathrm{mL}$. In summary, the sensor is linearly sensitive to the dielectric constant of solution at $1.9 \mathrm{GHz}$ and to the conductivity of solution at $2.1 \mathrm{GHz}$. These results show that the microwave hairpin resonator sensor can be applied to both dielectric liquid and aqueous $\mathrm{NaCl}$ detection. These stable linear relationships are advantageous for biodetection applications. Another advantage is the possibility of a planar structure, which is well suited for biosensor applications. Standard PCB fabrication technologies can be used to produce low-cost biosensing devices.

\section{Acknowledgments}

This study was financially supported by the National Science Council of Taiwan (under grant NSC 101-2221-E-006-004-MY3). We made use of shared facilities provided under the Program of Top 100 Universities Advancement funded by the Ministry of Education in Taiwan. The authors would like to thank the Center for Micro/Nano Science and Technology at National Cheng Kung University for granting us access to major equipment throughout the duration of this study and for their general technical support. 


\section{References}

1 A. Yúfera, A. Rueda, J. M. Muñoz, R. Doldán, G. Leger, and E. O. Rodríguez-Villegas: IEEE Trans. Circuits Syst. Regul. Pap. 52 (2005) 2620.

2 S. G. Badhe and S. N. Helambe: Sci. Res. Rep. 3 (2013) 239.

3 L. S. Jang and M. H. Wang: Biomed. Microdevices 9 (2007) 737.

4 Y. Cho, H. S. Kim, A. B. Frazier, Z. G. Chen, D. M. Shin, and A. Han: J. Microelectromech. Syst. 18 (2009) 808.

5 J. L. Hong, K. C. Lan, and L. S. Jang: Sens. Actuators, B 173 (2012) 927.

6 Y. C. Chuang, K. C. Lan, K. M. Hsieh, L. S. Jang, and M. K. Chen: Sens. Actuators, B 171-172 (2012) 1222.

7 G. Yoon: Biosens. Bioelectron. 26 (2011) 2347.

8 P. Mirtaheri, S. Grimnes, and Ø. G. Martinsen: IEEE Trans. Biomed. Eng. 52 (2005) 2093.

9 D. E. Yates, S. Levine, and T. W. Healy: J. Chem. Soc., Faraday Trans. 170 (1974) 1807.

10 T. Sun, N. G. Green, and H. Morgan: Nano 3 (2007) 55.

11 A. Peyman, C. Gabriel, and E. H. Grant: Bioelectromagnetics 28 (2007) 264.

12 J. Y. Jao, C. F. Liu, M. K. Chen, Y. C. Chuang, and L. S. Jang: Microelectron. Reliab. 51 (2011) 781.

13 M. Lohndorf, U. Schlecht, T. M. A. Gronewold, A. Malave, and M. Tewes: Appl. Phys. Lett. 87 (2005) 243902.

14 J. S. Hong, M. J. Lancaster, D. Jedamzik, and R. B. Greed: IEEE MTT-S Int. (IEEE, Baltimore, 1998) 367.

15 F. A. Miranda, G. Subramanyam, F. W. van Keuls, R. R. Romanofsky, J. D. Warner, and C. H. Mueller: IEEE Trans. Microwave Theory Tech. 48 (2000) 1181.

16 C. Dalmay, M. Cheray, A. Pothier, F. Lalloué, M. O. Jauberteau, and P. Blondy: Sens. Actuators, A 162 (2010) 189.

17 H. J. Lee, H. S. Lee, K. H. Yoo, and J. G. Yook: J. Appl. Phys. 108 (2010) 014908.

18 H. Melikyan, E. Danielyan, S. Kim, J. Kim, A. Babajanyan, J. Lee, B. Friedman, and K. Lee: Med. Eng. Phys. 34 (2012) 299.

19 O. Korostynska, M. Ortoneda-Pedrola, A. Mason, and A.I. Al-Shamma'a: Meas. Sci. Technol. 25 (2014) 065105.

20 R. W. Rhea: HF Filter Design and Computer Simulation (Noble Publishing, Atlanta, 1994).

21 J. S. Hong and M. J. Lancaster: Microstrip Filters for RF Microwave Applications (John Wiley \& Sons, New York, 2001).

22 M. Sagawa, K. Takahashi, and M. Makimoto: IEEE Trans. Microwave Theory Tech. 37 (1989) 1991.

23 S. Y. Lee and C. M. Tsai: IEEE Trans. Microwave Theory Tech. 48 (2000) 2482.

24 I. M. S. Lampreia, S. R. J. Magalhaes, S. I. M. Rodrigues, and A. F. S. S. Mendonca: J. Chem. Thermodyn. 38 (2006) 240.

25 L. Mayor, R. Moreira, F. Chenlo, and A. M. Sereno: J. Food Eng. 74 (2006) 253.

26 M. Ortoneda-Pedrola, O. Korostynska, A. Mason, and A. I. Al-Shamma'a: J. Phys. Conf. Ser. 450 (2013) 012016.

27 M. Ortoneda-Pedrola, O. Korostynska, A. Mason, and A. I. Al-Shamma'a: J. Phys. Conf. Ser. 450 (2013) 012013.

28 H. J. C. Blume, A. W. Love, M. J. van Melle, and W. W. Ho: IEEE J. Oceanic Eng. 2 (1977) 121.

29 R. P. Singh, V. Kumar, and S. K. Srivastav: Int. J. Remote Sens. 11 (1990) 321.

30 R. L. Smith Jr., S. B. Lee, H. Komori, and K. Arai: Fluid Phase Equilib. 144 (1998) 315.

31 M. Makimoto and S. Yamashita: Microwave Resonators and Filters for Wireless Communication (Springer, Berlin, 2000).

32 M. I. Khalil and S. I. Al-Resayes: Int. J. Phys. Sci. 7 (2012) 578.

\section{About the Authors}

Chia-Feng Liu is currently a Ph.D. student in the Department of Electrical Engineering, National Chen Kung University, Taiwan. He received his B.S. and M.S. degrees from the Department of Electrical Engineering, National Chen Kung University, Taiwan, in 2007 and 2010, respectively. His research focuses on the design and fabrication of microfluidic devices integrated with microwave resonators for single-cell measurement and analysis at radio frequencies. 
Ming-Kun Chen received his Bachelor's degree in electronic engineering from National Yunlin University of Science and Technology, Yunlin, Taiwan, in 1995, Master's degree in electronic engineering from I-Shou University, Kaohsiung, Taiwan, in 2002, and Ph.D. degree while at the Nondestructive Evaluation (NDE) Laboratory of the Electrical Engineering Department, National Cheng Kung University, Tainan, Taiwan, in 2006. From 1996 to 2002, he was at Advanced Semiconductor Engineering Test (ASET) Ltd., Kaohsiung, where he worked on IC testing interfaces and testing technology improvement. His research interests are in the areas of high-speed measurement, modeling, simulation methodology of packaging, the development of silicon sensor chips, microfluid handling systems for the measurement of living cells with microsensor systems, failure analysis of IC packaging/testing, analysis of signal integrity, and nondestructive testing.

Min-Haw Wang is currently an associate professor in the Department of Electrical Engineering at Chinese Culture University in Taiwan. He received his B.S. degree in electrical engineering at Chinese Culture University in 2000 and his M.S. degree in electrical engineering at National Tsing Hua University in 2003. He received his Ph.D. from the Department of Electrical Engineering, National Chen Kung University, Taiwan, in 2010. His current research focuses on microfluidics for biomedical applications.

Ling-Sheng Jang is currently a professor in the Department of Electrical Engineering at National Cheng Kung University in Taiwan. He received his B.S. degree in marine mechanical engineering at National Taiwan Ocean University in 1992 and his M.S. degree in mechanical engineering at National Chiao-Tung University in 1994. He received his Ph.D. in mechanical engineering from the University of Washington, Seattle, WA, in 2003. His current research focuses on microfluidics for biomedical applications. 